\title{
Decision making in resuscitation from out of hospital cardiac arrest
}

\author{
R Brown, E Jones, E Glucksman
}

\begin{abstract}
Objective-To determine which factors are perceived by senior house officers (SHOs), consultants, and medical registrars in accident and emergency (A\&E) medicine as being important in decision making.

Methods-132 SHOs in A\&E medicine, of 172 attending an induction course at the start of their job $(77 \%)$, completed a questionnaire relating to 20 factors of possible importance in decision making; 73 completed the questionnaire at six weeks and 55 at six months. Ten medical registrars and 31 consultants in A\&E medicine also completed the questionnaire.

Results-The SHOs were able to recognise bystander cardiopulmonary resuscitation and early advanced I support, as well as the presence of ventricular fibrillation, as important prognostic factors. There was considerable variation in all three groups in their opinions on the importance of the other factors considered. There was no obvious change in SHO responses over the period of training.

Conclusions-Lack of guidelines may result in more patients receiving resuscitation than are salvageable, as doctors maintain a low threshold for continuing resuscitation to avoid missing potential survivors. A decision making algorithm is recommended.

( $f$ Accid Emerg Med 1996;13:98-100)
\end{abstract}

Key terms: out of hospital; resuscitation; cardiac arrest

Chances of long term survival following out of hospital cardiac arrest are poor. ${ }^{12}$ The following factors are associated with a better prognosis: prompt bystander basic life support, ${ }^{3}$ short time to advanced cardiac life support, ${ }^{4}$ and the presence of ventricular fibrillation as the rhythm at arrest. ${ }^{5}$ However, the decision to continue resuscitation on arrival at hospital is frequently made by inexperienced junior medical staff who may be influenced by additional factors.

This project was designed to answer the following questions. (1) Which factors are perceived by senior house officers (SHOs) in accident and emergency ( $A \& E$ ) medicine as being important in decision making? (2) Does practical experience alter the perception of these factors? (3) Which factors are considered important by $A \& E$ consultants and medical registrars/senior registrars?

\section{Methods}

A questionnaire was constructed containing 20 diverse factors (fig 1). SHOs were asked to relate each factor to the decision to continue resuscitation after out of hospital cardiac arrest. The SHO was asked to rate each factor as important, not important, or of uncertain importance. The questionnaire was designed to include factors which have been shown to be important, as well as circumstances or conditions frequently perceived as relevant by junior doctors.

One hundred and seventy two A\&E SHOs from throughout the United Kingdom were given the questionnaire at an induction course at the start of the job. The same questionnaire was sent again at six weeks and at the end of the six month period. Forty randomly selected consultants in $A \& E$ and 10 medical registrars at King's College Hospital were also sent the questionnaire.

\section{Results}

Of the 172 SHOs who attended the induction course, 132 questionnaires were returned $(76 \%)$. Of 120 who gave a name and address, $73(60 \%)$ responded at six weeks, and 55 $(75 \%)$ of these replied at six months.

Of the 40 consultants contacted, $31(77 \%)$ replied and all 10 medical registrars returned a completed questionnaire.

Results are shown in the tables. The factors are grouped for clarity and for the purposes of discussion.

Ninety per cent of all respondents considered factors $1,2,12$, and 13 to be important. These are known to be of prognostic significance (table 1). Among the 55 SHOs who replied three times, there was no appreciable change in opinion over the six month period relating to these particular factors.

Factors 3, 4, 5, and 18 deal with the physical impression gained by initial inspection of the patient (table 2). Eighty per cent of SHOs and registrars thought age to be significant, although fewer $-65 \%$ and $52 \%$ respectively regarded obvious trauma as relevant. Trauma was judged important by $70 \%$ of the consultants. The body shape and condition was thought to be unimportant by $87 \%$ of all participants.

Ninety five per cent of SHOs and $80 \%$ of senior doctors felt that absence of pulse or respiratory effort was important; $87 \%$ of SHOs but less than half the registrars and consultants believed fixed and dilated pupils were important. 
Which of the following factors do you think are important in the decision to continue resuscitation from out of hospital cardiac arrest?

Answer: Important/Not important/Unsure

1. CPR started immediately after arrest

2. Short interval between arrest, ambulance response and arrival in A\&E

3. Age of patient

4. Body shape

5. Unkempt appearance

6. History of previous cardiac illness

7. Knowledge of other chronic illness

8. Presence of relatives

9. IV access established by ambulance crew with or without drugs administered

10. Intubated by ambulance crew

11. Defibrillation performed by ambulance crew

12. Rhythm on arrival of ambulance crew

13. Rhythm on arrival at hospital

14. Presence of pulse and/or respiratory effort

15. Fixed dilated pupils

16. Hypothermia

17. Patient vomited and possibly aspirated

18. Any obvious trauma

19. Advice of nursing staff/ambulance crew to continue resuscitation

20. Advice of nursing staff/ambulance crew not to continue resuscitation

Figure 1 Example of questionnaire

Factors 9, 10, and 11 are concerned with the type of treatment initiated by ambulance crews, and factors 19 and 20 with the interaction of the crew and other professionals with the doctor. Few of the SHOs (27\%) felt intravenous access was important, although $45 \%$ of the consultants felt it was important. The majority of SHOs, registrars, and consultants $(75 \%, 80 \%$, and $71 \%$ respectively) recognised that factor 11 , defibrillation performed by the crew, was important in its implication of the initial rhythm.

Information regarding previous history, which may affect quality of life in addition to prognosis for survival, was believed to be important by the majority of participants (table 3).

Table 1 Responses to factors 1, 2, 12, 13

\begin{tabular}{rlrl}
\hline & \multicolumn{2}{l}{ Considered important (\%) } \\
\cline { 2 - 4 } & SHO & Registrar & Consultant \\
\hline 1 & $96 \%$ & $100 \%$ & $90 \%$ \\
2 & $96 \%$ & $90 \%$ & $87 \%$ \\
12 & $78 \%$ & $80 \%$ & $87 \%$ \\
13 & $87 \%$ & $100 \%$ & $87 \%$ \\
\hline
\end{tabular}

Table 2 Responses to factors 3, 4, 5, 18

\begin{tabular}{rrrr}
\hline & \multicolumn{3}{l}{ Considered important/not important (\%) } \\
\cline { 2 - 4 } & SHO & Registrar & Consultant \\
\hline 3 & $80 / 03$ & $80 / 20$ & $68 / 10$ \\
4 & $11 / 82$ & $0 / 90$ & $13 / 81$ \\
5 & $2 / 89$ & $0 / 90$ & $3 / 90$ \\
18 & $65 / 24$ & $52 / 35$ & $70 / 30$ \\
\hline
\end{tabular}

Table 3 Responses to factors 6, 7, 8

\begin{tabular}{llll}
\hline & \multicolumn{3}{l}{ Considered important/not important (\%) } \\
\cline { 2 - 4 } & SHO & Registrar & Consultant \\
\hline 6 & $80 / 17$ & $40 / 40$ & $52 / 35$ \\
7 & $90 / 05$ & $80 / 20$ & $71 / 23$ \\
8 & $31 / 58$ & $20 / 70$ & $29 / 61$ \\
\hline
\end{tabular}

Over half the SHOs felt that advice offered by other health workers was important, although only $35 \%$ of the consultants agreed.

\section{Discussion}

The decision to continue resuscitation from out of hospital cardiac arrest is of necessity made quickly. The doctors questioned were not asked to identify where they generally made the decision but it is possible that the location may influence the decision making process. In order to facilitate the process it has been recommended that all patients arriving at A\&E departments should be assessed in the resuscitation room to allow the use of monitoring equipment. ${ }^{6}$

The results of our questionnaire show that there is a great variation in perception of the importance of 20 different factors, even among consultants. Clearly the lack of guidelines may result in more patients receiving resuscitation than are salvageable, as doctors maintain a low threshold for continuing resuscitation to avoid missing potential survivors. However, one must ask whether resuscitation is withheld from patients because of the same lack of guidelines.

Survival is related to speed of response and to the precipitating arrhythmia. ${ }^{7}$ The majority of SHOs recognised these to be important discriminators, even at the beginning of the six month job, presumably as a result of previous education.

Consideration of factors related to the initial impression of the patient produced remarkably similar results between all grades. While some investigators have found that age does not influence survival, others disagree. ${ }^{8}$ The majority of doctors replying felt age was important in decision making.

With the advent of advanced trauma life support and trauma teams, most arrests from trauma are considered potentially salvageable and aggressive resuscitation is performed. We would therefore expect factor 18 , the presence of obvious trauma, to be considered important in continuing resuscitation (unless there is a clearly lethal injury). The fact that only $65 \%$ of SHOs and just over $50 \%$ of consultants thought this important is therefore surprising. The explanation for this may be that the responders understood the questionnaire to be asking about primary cardiac arrest only, with coincidental minor trauma, rather than major trauma as a cause of cardiac arrest.

Information regarding previous history is often not available on arrival at the $A \& E$ department and resuscitation attempts should not be suspended while this information is obtained.

We included factor number 8 , the presence of relatives, as this may put pressure on individual doctors to continue on their behalf. Relatives may also influence the decision by their understanding of the situation, prior knowledge of the patients condition, or by personal wishes. However, less than a third felt this to be important in the decision making.

The interaction of the inexperienced junior doctor with ambulance paramedics and 


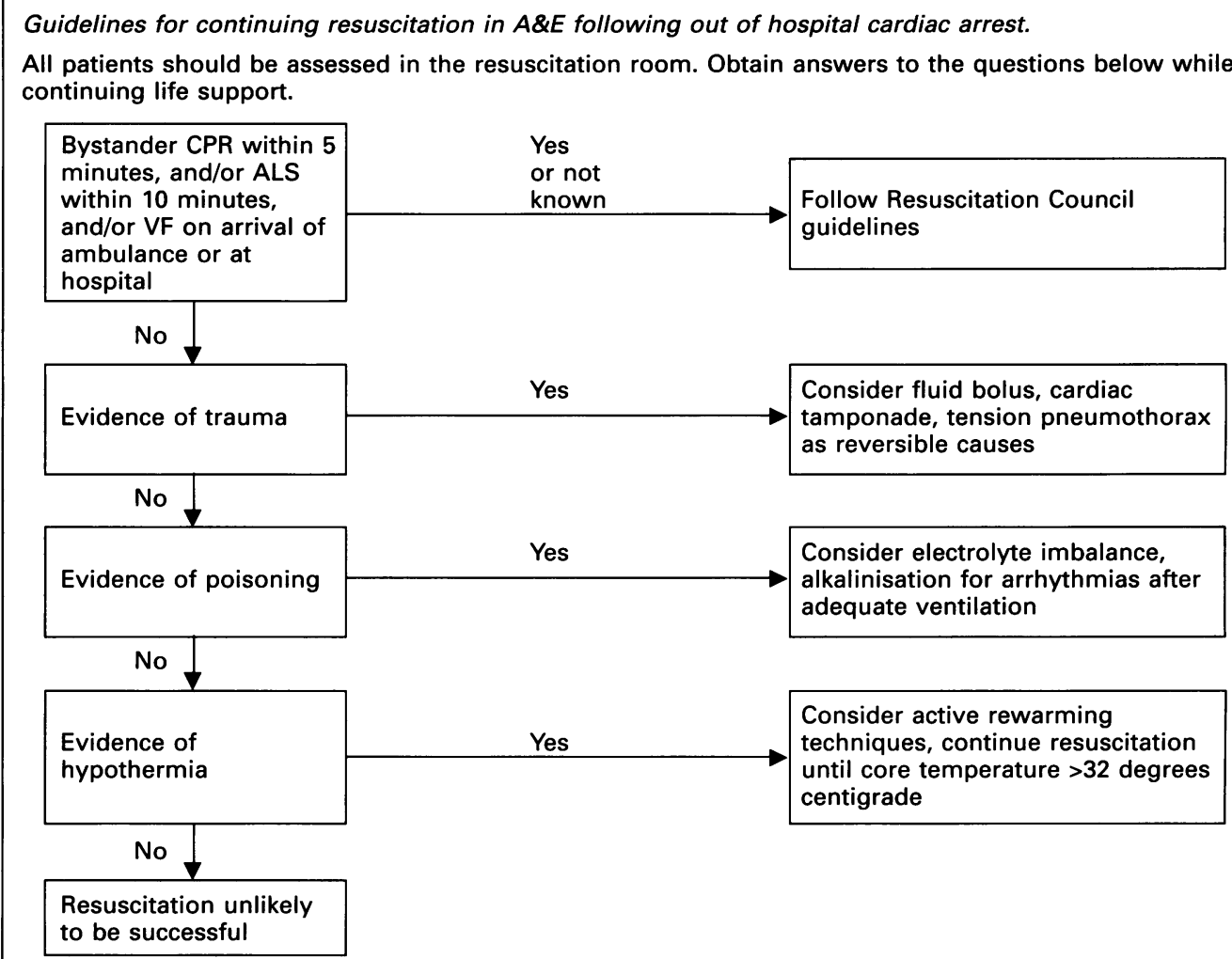

Figure 2 Guideline algorithm

experienced nursing staff is explored in factors $9,10,11,19$, and 20 . We expected the response to factors 19 and 20 to change with experience but there was little difference in the distribution of responses.

Overall there was great variation among answers and this may reflect different departmental policies and practice. Although competence and confidence in resuscitation skills would be expected to increase with the quantity of resuscitation experience, no attempt was made to correlate replies with the number of arrests attended, or number of cases where the SHO had made a decision. We have no way, therefore, of identifying the precise amount of experience obtained. Nevertheless, there is a small decrease in the total number of unsure responses $(6 \cdot 5 \%$ to $4 \cdot 5 \%)$.

\section{CONCLUSIONS}

In the questionnaire there are three factors which have previously been shown to be of prognostic significance. SHOs are able to identify the importance of these factors correctly, even at the start of the job. However, there is wide variation between the responses to all other factors, even among more experienced doctors. This would indicate that SHOs are influenced by factors which may well be of value in deciding further treatment options, but should not be sought or considered until the primary decision to continue has been made.

Additionally, in-post training and experience do not appear to clarify the situation for the juniors, which is reflected in the variability of opinion among the registrars and consultants questioned.

The lack of established guidelines or simple algorithms for the purposes of distinguishing salvageable patients may lead to confusion and unhappiness among junior staff faced with making difficult decisions.

Using the three established variables known to influence outcome, we have constructed a simple prompt to facilitate the decision making process in the circumstances of continuing resuscitation in $A \& E$ from out of hospital cardiac arrest (fig 2).

1 Eliastam M, Duralde T, Martinez F, Schwartz D. Cardiac arrest in the emergency medical service system: guidelines for resuscitation. $f \mathrm{Am}$ Coll Emerg Physicians 1977; 6:525-9.

2 Gray WA, Capone RJ, Most AS. Unsuccessful emergency medical resuscitation; are continued efforts in the Emergency Department justified? N Engl f Med 1991; 325:1393-8.

3 Cummins RO, Eisenberg MS, Hallstrom AP, Litwin PE. Survival of out-of-hospital cardiac arrest with early initiation of cardiopulmonary resuscitation. $\mathrm{Am} \mathcal{F}$ Emerg Med 1985;3:114-8.

4 Eisenberg MS, Berner L, Hallstrom A. Survivors of out-ofhospital cardiac arrest: morbidity and long-term survival. Am $\mathcal{f}$ Emerg Med 1984;2:189-92.

5 Aprahamian C, Thompson BM, Gruchow HW, Mateer JR, Tucker JF, Stueven HA, et al. Decision making in prehospital sudden cardiac arrest. Ann Emerg Med 1986;15:445-9.

6 Christian MS, Gosnold JK, Kersley PN. Confirmation of death. $B M \mathcal{F}$ 1980;281:717-9.

7 Eisenberg M, Hallstrom A, Bergner L. The ACLS score: predicting survival from out-of-hospital cardiac arrest. fAMA 1981;246:50-2.

8 Tresch DD, Thakur RK, Hoffinan RG, Olson D, Brooks HL. Should the elderly be resuscitated following out-ofhospital cardiac arrest? Am $\mathcal{F}$ Med 1989;86:145-50. 